\title{
Öber die Bindung von Kohlensäure durch Alkohole, Zucker und Oxysäuren.
}

\author{
Von \\ M. Siegfried und S. Howwjanz.
}

(Aus der chemischen Abteilung des physiologischen Instituts der Universität Leipzig.) (Der Redaktion zugegangen am 31. März 1909.)

Bei Ausarbeitung der Methode der Bestimmung des Quotienten $\frac{\mathrm{CO}_{2}}{\mathrm{~N}}$ bei der Carbaminoreaktion wurde gefunden, ${ }^{1}$ ) daß man für diesen Quotienten etwas zu große Werte erhält, wenn man die Reaktion bei Gegenwart selbst sehr geringer Mengen Alkohol, z. B. einiger Tropfen alkoholischer Phenolphthaleinlösung vornimmt. Dies beruht auf der Tatsache, daß Alkohol ebenso wie Aminokörper Kohlensäure zu binden vermag, wenn die Gelegenheit vorhanden ist, da $\beta$ die entstehende Carbonsäure in ein Salz, z. B. in das Kalksalz übergeführt wird. Ebenso wie Äthylalkohol verhalten sich andere Alkohole, Zuckerarten und Oxysäuren. ${ }^{2}$ ) Diese Reaktion ist als Hydroxylkohlensäurereaktion in der vorläufigen Mitteilung bezeichnet worden.

Der Verlauf dieser Reaktion wird auf S. 394 ff. diskutiert werden.

Die physiologische Bedeutung der Reaktion ergibt sich, wenn man bedenkt, daß Hydroxylkörper dieselbe Funktion, wie die Aminokörper verrichten können, unter Berücksichtigung der früher ausgeführten Konsequenzen. ${ }^{3}$ ) Über eine weitere Anwendung, welche die Hydroxylkohlensäure- und die Carbaminoreaktion im tierischen und pflanzlichen Organismus erfahren können, wird weiter unten (S. 404) gesprochen werden. Die Untersuchung, die zunächst darauf zielte, Grundlagen für die Reaktion als biologisch interessante zu gewinnen, hat auch

1) M. Sieg fried, Diese Zeitschrift, Bd. LII, S. 506 .

") M. Siegfried u. C. Ne umann, Diese Zeitschrift, Bd. LIV, S. 428.

3) M. Siegfried, Diese Zeitschrift, Bd. XLIV, S. 95.

M. Sieg fried u. H. Li e bermann, Diese Zeitschrift, Bd.LIV, S. 4 141. 
Ergebnisse geliefert, welche für die sogenannte reine Chemie beachtenswert erscheinen.

\section{Die Methode.}

Ähnlich wie bei der Carbaminoreaktion handelt es sich hier darum, die Menge der unter möglichst gleichen Bedingungen von Hydroxylkörpern aufnehmbaren und abspaltbaren Kohlensäure zu bestimmen. Weil die hydroxylkohlensauren Salze viel leichter zersetzlich sind als die aminokohlensauren, erfordert die Ausführung der Methode die Einhaltung möglichst genau bei $0^{0}$ liegender Temperaturen. Auch die Bestimmung der in Reaktion getretenen Mengen der Hydroxylkörper ist umständlicher und nicht so scharf, als die Bestimmung der Mengen der Aminokörper bei der Carbaminoreaktion durch die Ermittelung des Stickstoffes nach Kjeldahl.

\section{A. Der Quotient.}

Der Quotient: $\frac{\mathrm{CO}_{2}}{\mathrm{~S}}$

gibt das Verhältnis der molekularen Menge Kohlensäure zu der molekularen Menge der Substanz an, d. h. er zeigt, wieviel Moleküle Kohlensäure von 1 Molekül der Substanz aufgenommen und abgespalten wird.

Wenn b g Substanz nach Behandeln mit Kohlensäure bei Gegenwart überschüssigen Kalkhydrates a g Calciumcarbonat abspalten, so ist der Quotient $\frac{\mathrm{a}: 100}{\mathrm{~b}: \mathrm{M}}$, da $100(100,1)$ das Molekulargewicht des Calciumcarbonates ist, und 1 Molekül aufgenommener Kohlensäure 1 Molekül abgespaltenen Calciumcarbonates entspricht, und wenn $M$ das Molekulargewicht der betreffenden Substanz bedeutet.

Beispiel: 0,2000 g Methylalkohol gaben 0,3755 $\mathrm{g} \mathrm{CaCO}_{3}$, Molekulargewicht des Methylalkohols $=32$

$$
\text { Quotient } \frac{\mathrm{CO}_{2}}{\mathrm{~S}}=\frac{0,3755: 100}{0,2000: 32}=0,601
$$

d. h. 1 Molekül Methylalkohol nimmt 0,601 Molekül $\mathrm{CO}_{2}$ auf. 
B. Die Bestimmung der Menge des Hydroxylkörpers.

\section{Bei optisch inaktiven Substanzen.}

Bei optisch inaktiven Substanzen werden die in Reaktion tretenden Mengen durch Wägung vor dem Versuche bestimmt. Es ist in diesem Falle notwendig, nach der Behandlung mit Kohlensäure den Kalkniederschlag auszuwaschen, um die ganze Menge des hydroxylkohlensauren Salzes im Filtrate, aus dem dann das Calciumcarbonat abgeschieden wird, zu erhalten. Die angestellten Versuche ergaben, daß $100 \mathrm{ccm}$ eiskalten Kalkwassers zum Auswaschen genügen, wenn zum Absaugen eine Filterplatte von $4 \mathrm{~cm}$ verwendet wurde. Folgendes Beispiel zeigt dies.

a) Der Niederschlag wurde nicht ausgewaschen:

$\begin{array}{ccc} & \text { Angew. g Äthylalkohol } & \text { Quotient } \\ 1 . & 0,15 & 0,132 \\ 2 . & 0,20 & 0,145 \\ 3 . & 0,25 & 0,139\end{array}$

b) Der Niederschlag wurde mit $100 \mathrm{ccm}$ eiskalten Kalkwassers ausgewaschen:

$\begin{array}{lll}1 . & 0,15 & 0,171 \\ 2 . & 0,20 & 0,189 \\ 3 . & 0,25 & 0,170\end{array}$

c) Der Niederschlag wurde mit $200 \mathrm{ccm}$ eiskalten Kalkwassers gewaschen:

$\begin{array}{lll}1 . & 0,15 & 0,170 \\ 2 . & 0,20 & 0,179\end{array}$

Wenn auch der Quotient sich bei weiterem Auswaschen nicht mehr verändert, so besteht doch die Möglichkeit, daß sich im Niederschlage unlösliche Kalksalze von Hydroxylkohlensäureverbindungen befinden. Dies findet tatsächlich bei mehrwertigen Alkoholen bis zu einem geringen Grade statt. Durch Zersetzen der Kalkniederschläge mit warmem Wasser und Ammoniumcarbonat ließen sich auch aus den ausgewaschenen Niederschlägen geringe Mengen der Alkohole regenerieren.

So wurden, um Beispiele anzuführen, bei denen die zurückgehaltenen Mengen Substanz besonders groß waren, bei Verwendung von $0,8988 \mathrm{~g}$ Mannit aus dem Kalkniederschlage 0,1032 g Mannit wiedergewonnen. Die Menge Mannit, der die 
gefundene Menge Calciumcarbonat entspricht, betrug also $0,8988-0,1032 \mathrm{~g}=0,7956 \mathrm{~g}$. Da $0,6850 \mathrm{~g} \mathrm{CaCO}_{3}$ erhalten wurden, ist der Quotient $\frac{\mathrm{CO}_{2}}{\mathrm{~S}}$ ohne Berücksichtigung der aus dem Kalkniederschlage regenerierten Menge Mannit $=1,39$ mit Berücksichtigung derselben $=1,56$.

So wurden ferner bei Anwendung von 0,4497 g Dulcit aus dem Kalkniederschlage $0,0650 \mathrm{~g}$ Dulcit wiedergewonnen. $\mathrm{CaCO}_{3}$ war gefunden: 0,3181 g. Der Quotient ist also ohne Korrektur: 1,30 und mit Korrektur: 1,50. Es wäre daher bei diesen Körpern der Kalkniederschlag zu zersetzen und die wiedergewonnene Substanz von der angewandten abzuziehen, wenn man annimmt, daß die regenerierte Substanz im Niederschlage als solche, nicht als Kohlensäureverbindung vorhanden ist. Ist aber das letztere der Fall, was wahrscheinlich ist, so würde ja, wenn die zurückgehaltene Menge der Substanz in das Filtrat übergegangen wäre, auch nachher Calciumcarbonat in der dieser Menge entsprechenden Quantität entstanden sein. Es wäre also unrichtig, die Korrektur zu machen. Deshalb ist die Korrektur nicht vorzunehmen. Man hat sich aber in jedem Falle zu überzeugen, daß die Menge der im Kalkniederschlage zurückgehaltenen Substanz nicht zu groß ist, etwa 10\% übersteigt. Im ungünstigsten Falle würde dann ein Fehler von $10 \%$ gemacht. Aus den eben angeführten Gründen ist jedoch der Fehler voraussichtlich sehr gering.

\section{Bei optisch aktiven Substanzen.}

Bei den optisch aktiven Substanzen wird die in Reaktion getretene Substanzmenge aus dem Filtrate vom Kalkniederschlage durch Polarisation ermittelt. Bei den Zuckern, welche hier in erster Linie in Frage kommen, ist es erforderlich, das Filtrat vom Kalkniederschlage auf ein bestimmtes Volumen $(200 \mathrm{ccm})$ im Maßkolben aufzufüllen, die Hälfte zur Bestimmung des abspaltbaren Calciumcarbonates, die andere Hälfte zur Polarisation zu verwenden. Bei Zuckern, welche eine Birotation besitzen, muß die Zuckerlösung vor Anstellung der Hydroxylkohlensäurereaktion so lange stehen, bis erfahrungsgemäß die konstante 
Drehung eingetreten ist, da sich die labilen Modifikationen der Zucker möglicherweise anders gegen Kohlensäure als die stabilen Modifikationen verhalten.

Ehe die Substanzmenge in der Hälfte des Filtrates vom Kalkniederschlage durch Polarisation bestimmt werden kann, muß die Lösung neutralisiert bezw. schwach angesäuert werden. Dies hat durch tropfenweisen Zusatz von ca. 3\%iger Essigsäure zu geschehen. Ein geringer Überschuß derselben beeinflußt die spezifische Drehung nicht, wohl aber ein größerer sowie ein geringer Überschuß von Salzsäure, wie durch vergleichende Versuche auch bei Hexosen festgestellt wurde. Selbstverständlich hat der Zusatz von Essigsäure aus der Bürette zu geschehen, denn das Volumen der zugesetzten Essigsäure verringert die Konzentration an aktiver Substanz und muß daher in Rechnung gezogen werden.

\section{Die Ausführung der Methode.}

Die wässerige Lösung des Hydroxylkörpers (in der Regel $50 \mathrm{ccm}$ ) wird in einer ca. $300 \mathrm{ccm}$ fassenden Glasbüchse in einer schwachen Kältemischung soweit abgekühlt, daß eben etwas Eis aus der Lösung krystallisiert. Dazu wird von $50 \mathrm{ccm}$ in Eiswasser abgekühlter Kalkmilch (aus $150 \mathrm{~g}$ Calciumoxyd aus Marmor und 1 Liter Wasser bereitet) ca. $10 \mathrm{ccm}$ und einige Tropfen einer Lösung von Phenolphthalein in Kalkwasser gegeben; es wird kräftig geschüttelt, Kohlensäure bis fast zum Verschwinden der Färbung eingeleitet, wieder etwa $10 \mathrm{ccm}$ Kalkmilch dazugegeben, wieder Kohlensäure wie das erstemal eingeleitet, nochmals etwa $10 \mathrm{ccm}$ Kalkmilch dazugegeben, wieder kräftig geschüttelt, wieder Kohlensäure eingeleitet, hierauf der Rest, etwa $20 \mathrm{ccm}$ Kalkmilch, dazugegeben und kräftig geschüttelt. Während der ganzen Operation wird in der Kältemischung abgekühlt, sodaß sich eine kleine Menge Eis in der Reaktionsmischung befindet. Hierauf wird auf einer Saugplatte von $4 \mathrm{~cm}$ Durchmesser abgesaugt. Der Trichter ist durch den Hals einer weiten Glocke geführt und in dieser mit Kältemischung umgeben. Ebenso befindet sich der Saugkolben in einer Kältemischung. Der Kalkniederschlag wird 
a) bei Hydroxylkörpern, welche optisch inaktiv sind, mit $100 \mathrm{ccm}$ eisgekühlten Kalkwassers ausgewaschen;

b) bei Hydroxylkörpern, welche optisch aktiv sind, nicht ausgewaschen.

1. Bei optisch inaktiven Körpern, welche sich nicht beim Erhitzen ihrer wässerigen Lösungen zersetzen, wird das Filtrat mit dem gleichen Volumen ausgekochten Wassers verdünnt (da Kalkhydrat in heißem Wasser schwerer löslich ist, als in kaltem) und in einem mit nach abwärts gebogenen Natronkalkrohr versehenen Erlenmeyer zum Sieden erhitzt; nach dem Abkühlen wird das gebildete Calciumcarbonat auf gewogenem Gooch-oder Neubauer-Tiegel abgesaugt, mit Wasser gewaschen, bei $120^{\circ}$ getrocknet und gewogen.

2. Bei optisch inaktiven Körpern, welche sich beim Erhitzen der wässerigen Lösungen verändern, wird wie unter a verfahren mit der Abänderung, daß das Filtrat nicht erhitzt, sondern zur vollständigen Abscheidung der abspaltbaren Kohlensäure als Calciumcarbonat 12 bis 16 Stunden bei gewöhnlicher Temperatur stehen gelassen wird. In diesem Falle ist die Verdünnung mit ausgekochtem Wasser nicht erforderlich.

3. Bei optisch aktiven Substanzen wird das Filtrat in einen $100 \mathrm{ccm}$ fassenden Maßkolben übergeführt und bis zur Marke mit ausgekochtem Wasser aufgefüllt. Die Hälfte der gut durchgeschüttelten Lösung, in der sich bereits Calciumcarbonat abgesetzt hat, wird in einem mit Natronkalkrohr geschützten Erlenmeyer bei gewöhnlicher Temperatur 12 bis 16 Stunden stehen gelassen, dann das ausgeschiedene Calciumcarbonat auf gewogenem Gooch-oder Neubauer-Tiegel abgesaugt, mit Wasser gewaschen, bei $120^{\circ}$ bis zum konstanten Gewichte getrocknet und gewogen.

Die andere Hälfte der Mischung wird sogleich mit 3\%oiger Essigsäure aus der Bürette schwach angesäuert und im $4 \mathrm{dm}$ Rohr polarisiert. Aus dem spezifischen Drehungsvermögen und dem gefundenen Winkel $\alpha$ wird unter Berücksichtigung der durch den Essigsäurezusatz bewirkten Verdünnung die dem gefundenen Calciumcarbonat entsprechende Menge des Hydroxyl- 
körpers ermittelt. Die Berechnung des Quotienten geschieht nach S. 377.

\section{Ergebnisse.}

A. Einwertige Alkohole.

\section{Methylalkohol.}

Molekulargewicht : 32 . Sdp. gefunden: $65-66^{\circ}$. $2,5000 \mathrm{~g}$ Methylalkohol wurden zu $50 \mathrm{ccm}$ gelöst.

Hiervon wurden die in der Tabelle angegebenen Kubikzentimeter mit $50 \mathrm{ccm}$ Wasser verdünnt zur Reaktion verwendet.

\begin{tabular}{|c|c|c|c|c|}
\hline $\begin{array}{l}\text { ccm der } \\
\text { Lösung }\end{array}$ & $\begin{array}{l}\text { Methyl- } \\
\text { alkohol } \\
\mathrm{g}\end{array}$ & $\begin{array}{c}\mathrm{CaCO}_{3} \\
\mathrm{~g}\end{array}$ & Quotient & $\begin{array}{c}\text { Quotient im } \\
\text { Mittel }\end{array}$ \\
\hline 1 & 0,05 & $0,082 \check{5}$ & 0,526 & \\
\hline 1 & 0,05 & 0,0800 & 0,513 & 0,566 \\
\hline 1 & 0,05 & 0,1028 & 0,660 & \\
\hline 2 & 0,1 & 0,2061 & 0,658 & \\
\hline 2 & 0,1 & 0,1640 & 0,524 & 0,613 \\
\hline 2 & 0,1 & 0,2060 & 0,658 & \\
\hline 3 & 0,15 & 0,2814 & 0,599 & \\
\hline 3 & 0,15 & 0,2859 & 0,610 & 0,591 \\
\hline 3 & 0,15 & 0,2650 & 0,565 & \\
\hline 4 & 0,2 & 0,3755 & 0,601 & \\
\hline 4 & 0,2 & 0,3492 & 0,558 & 0,586 \\
\hline 4 & 0,2 & 0,3748 & 0,600 & \\
\hline o & 0,25 & 0,5140 & 0,657 & \\
\hline 5 & 0,25 & 0,4652 & 0,595 & 0,588 \\
\hline 5 & 0,25 & 0,4010 & 0,513 & \\
\hline 6 & 0,3 & 0,5443 & 0,581 & \\
\hline 6 & 0,3 & 0,6529 & 0,697 & 0,634 \\
\hline 6 & 0,3 & 0,5879 & 0,626 & \\
\hline \multicolumn{4}{|c|}{ Quotient Gesamtmittel . . } & 0,596 \\
\hline
\end{tabular}


Wie man sieht, ist die Genauigkeit der einzelnen Bestimmungen keineswegs so groß wie die der Bestimmungen des Quotienten der Carbaminoreaktion. Dies ist in erster Linie dadurch bedingt, daß hier das Gleichgewicht der Reaktion(s. S. 401) bereits verschoben ist, sobald der Kalkniederschlag mit dem Calciumcarbonat abfiltriert ist.

Jedoch die Mittel aus drei hintereinander ausgeführten Bestimmungen weichen vom Gesamtmittel nur wenig ab. Diese Abweichung beträgt:

1. Bei Verwendung von $0,05 \mathrm{~g}$ Methylalkohol $5 \%$ vom Gesamtmittel

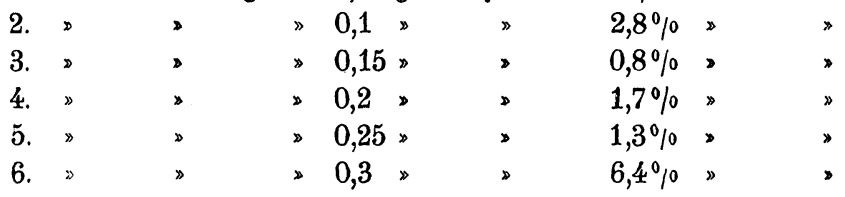

Die Konzentration des Methylalkohols innerhalb der eingehaltenen Grenzen ist nicht von Einfluß auf die Größe des Quotienten. Bei Versuchen, bei denen die Reaktion einige Grade über 0 vorgenommen wurde, wurde hingegen gefunden, daß auch innerhalb dieser Grenzen die Konzentration den Quotienten beeinflußt. Hierauf soll später eingegangen werden.

\section{2. Äthylalkohol.}

Molekulargewicht: 46. Sdp. gefunden $78^{\circ}$.

$\mathrm{Zu}$ jedem Versuche waren $4 \mathrm{ccm}$ einer $5 \%$ igen Lösung mit $50 \mathrm{ccm}$ Wasser verdünnt verwendet worden.

\begin{tabular}{c|c|c|c}
\hline \hline $\mathrm{g}$ Äthylalkohol & $\mathrm{g} \mathrm{CaCO}_{3}$ & Quotient & Quotient im Mittel \\
\hline 0,2 & 0,0756 & 0,175 & \\
0,2 & 0,0826 & 0,189 & 0,184 \\
0,2 & 0,0744 & 0,170 & \\
0,2 & 0,0868 & 0,200 &
\end{tabular}

\section{Propylalkohol.}

Molekulargewicht: 60 . Sdp. gefunden $95^{\circ}$.

Je $4 \mathrm{ccm}$ einer $5 \%$ igen Lösung mit $50 \mathrm{ccm}$ Wasser. 


\begin{tabular}{c|c|c|c}
\hline \hline g Propylalkohol & $\mathrm{g} \mathrm{CaCO}_{3}$ & Quotient & Quotient im Mittel \\
\hline 0,2 & 0,0670 & 0,201 & \\
0,2 & 0,0640 & 0,192 & 0,190 \\
0,2 & 0,0598 & 0,180 & \\
0,2 & 0,0627 & 0,186 &
\end{tabular}

4. Butylalkohol.

Molekulargewicht: 74. Sdp. gefunden 115-116 ${ }^{\circ}$.

Je $4 \mathrm{ccm}$ einer $5 \%$ igen Lösung mit $50 \mathrm{ccm}$ Wasser.

\begin{tabular}{c|c|c|c}
\hline g Butylalkohol & $\mathrm{g} \mathrm{CaCO}_{3}$ & Quotient & Quotient im Mittel \\
\hline 0,2 & 0,0569 & 0,211 & \\
0,2 & 0,0608 & 0,222 & 0,207 \\
0,2 & 0,0540 & 0,200 & \\
0,2 & 0,0526 & 0,196 &
\end{tabular}

\section{Isobutylalkohol.}

Molekulargewicht: 74. Sdp. gefunden 106-107\%.

Je $4 \mathrm{ccm}$ einer $5 \%$ igen Lösung mit $50 \mathrm{ccm}$ Wasser.

\begin{tabular}{c|c|c|c}
\hline \hline g Isobutylalkohol & $\mathrm{g} \mathrm{CaCO}_{3}$ & Quotient & Quotient im Mittel \\
\hline 0,2 & 0,0593 & 0,218 & \\
0,2 & 0,0552 & 0,204 & 0,194 \\
0,2 & 0,0490 & 0,181 & \\
0,2 & 0,0473 & 0,174 &
\end{tabular}

6. Tertiärer Butylalkohol.

Molekulargewicht: 74. Sdp. gefunden $82^{\circ}$.

Je $4 \mathrm{ccm}$ einer $5 \%$ igen Lösung mit $40 \mathrm{ccm}$ Wasser.

\begin{tabular}{c|c|c|c}
\hline g tert. Butylalkohol & $\mathrm{g} \mathrm{CaCO}_{3}$ & Quotient & Quotient im Mittel \\
\hline 0,2 & 0,0065 & 0,026 & \\
0,2 & 0,0086 & 0,033 & 0,034 \\
0,2 & 0,0100 & 0,037 & \\
0,2 & 0,0106 & 0,041 &
\end{tabular}


Über die Bindung von Kohlensäure durch Alkohole usw.

\section{Benzylalkohol.}

Molekulargewicht: 108. Sdp. gefunden $204^{\circ}$.

Je $10 \mathrm{ccm}$ einer $2 \%$ igen Lösung mit $40 \mathrm{ccm}$ Wasser.

\begin{tabular}{c|c|c|c}
\hline $\begin{array}{c}\text { Benzylalkohol } \\
\mathbf{g}\end{array}$ & $\begin{array}{c}\mathrm{CaCO}_{3} \\
\mathrm{~g}\end{array}$ & Quotient & Quotient im Mittel \\
\hline 0,2 & 0,1178 & 0,638 & \\
0,2 & 0,1197 & 0,650 & 0,652 \\
0,2 & 0,1154 & 0,622 & \\
0,2 & 0,1289 & 0,697 &
\end{tabular}

Die Versuche zeigen, daß die einwertigen Alkohole ein ausgesprochenes Vermögen besitzen, Kohlensäure zu binden. Zweifellos (s. S. 394) entstehen hier die Salze der Alkylkohlensäuren. Die Salze dieser sauren Ester bilden sich bei $0^{0} \mathrm{z}$. B. beim Methylalkohol in ca. 0,1\% igen Lösungen bis zu 60\%! Es fällt sofort der große Unterschied zwischen diesem Alkohol und seinen Homologen auf. 1 Molekül Methylalkohol bindet unter gleichen Verhältnissen ca. dreimal so viel Kohlensäure als Äthylalkohol, Propylalkohol, normaler und Isobutylalkohol. Ebenso wie Methylalkohol verhält sich der Benzylalkohol. Fast gar nicht oder gar nicht reagiert der tertiäre Butylalhohol.

Wir sehen hier einen ausgesprochenen Parallelismus zwischen dem Verhalten der Alkohole gegen Kohlensäure und der Esterifikationsgeschwindigkeit dieser Alkohole. Nach Mentschutkin $^{1}$ ) ist die Anfangsgeschwindigkeit der Veresterung mit Essigsäure beim Äthylalkohol, Propylalkohol normalem Butylalkohol fast gleich, die des Methylalkohols erheblich größer, die des tertiären Butylalkohols sowie anderer tertiärer Alkohole außerordentlich gering.

B. Mehrwertige Alkohole.

\section{1. Äthylenglykol.}

Molekulargewicht: 62 .

$\mathrm{Zu}$ jedem Versuche wurden $50 \mathrm{ccm}$ einer einprozentigen

1) N. Mentschutkin, Lieb. Ann., Bd. CXCV, S. 334 u. Bd. GXCVII, S. 193. 
wässerigen Lösung im Vakuum destillierten Äthylenglykols Kahlbaum verwendet.

\begin{tabular}{|c|c|c|c|c|}
\hline & $\begin{array}{c}\text { Äthylenglykol } \\
\mathrm{g}\end{array}$ & $\begin{array}{c}\mathrm{CaCO}_{3} \\
\mathrm{~g}\end{array}$ & Quotient & Quotient im Mittel \\
\hline 1. & 0,5 & 0,8682 & 1,075 & \multirow{2}{*}{1,05} \\
\hline 2. & 0,5 & 0,8262 & 1,022 & \\
\hline
\end{tabular}

2. Glycerin.

Molekulargewicht: 92.

Das Glycerin wurde im Vakuum in 4 gewogene Vorlagen destilliert, der Inhalt der einzelnen Vorlagen nach Wägung in Maßkolben übergespült und mit Wasser aufgefüllt. Zu jedem Versuche dienten $50 \mathrm{ccm}$ der Lösung.

\begin{tabular}{l|c|c|c|c}
\hline \hline & $\begin{array}{c}\text { Glycerin } \\
\mathrm{g}\end{array}$ & $\begin{array}{c}\mathrm{CaCO}_{3} \\
\mathrm{~g}\end{array}$ & Quotient & Quotient im Mittel \\
\hline & & 1,0000 & 0,935 & \\
2. & 0,9833 & 1,1062 & 1,034 & \\
3. & 0,9833 & 1,1225 & 1,051 & \\
$\mathbf{4 .}$ & 0,9833 & 1,0958 & 0,942 & \\
5. & 0,0698 & 1,1762 & 1,009 & 1,025 \\
6. & 0,0698 & 1,1296 & 0,989 & \\
7. & 0,0698 & 0,6620 & 1,239 & \\
8. & 0,4914 & 0,5849 & 1,002 & \\
& 0,5349 & & &
\end{tabular}

3. Erythrit.

Molekulargewicht: 122. Fp. gefunden $120^{\circ}$.

\begin{tabular}{l|c|c|c|c}
\hline \hline & $\begin{array}{c}\text { Erythrit } \\
\mathrm{g}\end{array}$ & $\begin{array}{c}\mathrm{CaCO}_{8} \\
\mathrm{~g}\end{array}$ & Quotient & Quotient im Mittel \\
\hline & & & & \\
2. & 0,5554 & 0,5735 & 1,261 & \\
2. & 0,6832 & 0,7326 & 1,308 & \\
3. & 0,7126 & 0,5878 & 1,012 & 1,16 \\
4. & 0,5000 & 0,4322 & 1,053 &
\end{tabular}

Aus den Kalkniederschlägen der Versuche 3 und 4 wurde Erythrit regeneriert. Die Menge betrug nur: bei 3. 0,0254 g, bei 4. $0,0330 \mathrm{~g}$, sodaß sich nach Abzug dieser Mengen von 
der angewandten Substanz der Quotient des dritten Versuches von 1,012 auf 1,044, der des vierten Versuches von 1,053 auf 1,131 erhöht. Diese Differenzen sind belanglos; man ist aber nach S. 397 nicht einmal berechtigt, diese Korrektion zu machen.

\section{Quercit.}

Molekulargewicht: 164.

$1,5017 \mathrm{~g} \mathrm{zu} 25 \mathrm{ccm}$ gelöst; gef.: $\alpha=3,08^{\circ}$ bei l $=2$. T. 20 .

$\alpha_{[D]}{ }^{20}=25,63^{\circ}$ anstatt $24,16^{\circ}$. Zur Berechnung der Substanzmenge ist in folgenden Versuchen $\alpha_{[D]}=24,16^{\circ}$ genommen.

In diesem Falle wurden die Filtrate auf $100 \mathrm{ccm}$ aufgefüllt, $\alpha$ und $\mathrm{CaCO}_{3}$ in je $50 \mathrm{ccm}$ bestimmt.

1. $\alpha=0,17^{\circ} \mathrm{l}=2$ Essigsäure korrigiert $1 \mathrm{ccm} ; \mathrm{CaCO}_{3}$ $=0,1140 \mathrm{~g}$, Quotient $=1,046$.

2. $\alpha=0,45 \mathrm{l}=2$ Essigsäure korrigiert $1,2 \mathrm{ccm} ; \mathrm{CaCO}_{3}$ $=0,2778 \mathrm{~g}$, Quotient $=0,995$.

Quotient im Mittel $=1,02$.

\section{Mannit.}

Molekulargewicht: 182 . Fp. gefunden $165-166^{\circ}$.

\begin{tabular}{l|c|c|c|c}
\hline & $\begin{array}{c}\text { Mannit } \\
\mathbf{g}\end{array}$ & $\begin{array}{c}\mathrm{CaCO}_{\mathbf{3}} \\
\mathbf{g}\end{array}$ & Quotient & Quotient im Mittel \\
\hline 1. & 0,8988 & 0,6850 & 1,385 & \\
2. & 0,8062 & 0,6119 & 1,381 & 1,415 \\
3. & 0,4040 & 0,3284 & 1,479 &
\end{tabular}

In allen drei Versuchen wurde der Kalkniederschlag mit Ammoniumcarbonat zersetzt und aus ihm Mannit regeneriert. Es wurden erhalten: $1.0,1032 \mathrm{~g}, 2.0,0800 \mathrm{~g}, 3.0,0397 \mathrm{~g}$.

\section{Dulcit.}

Molekulargewicht 182. Fp. gef. $187^{\circ}$.

\begin{tabular}{c|c|c|c|c}
\hline \hline & $\begin{array}{c}\text { Dulcit } \\
\mathbf{g}\end{array}$ & $\begin{array}{c}\mathrm{CaCO}_{\mathbf{3}} \\
\mathbf{g}\end{array}$ & Quotient & Quotient im Mittel \\
\hline 1. & 0,4497 & 0,3181 & 1,287 & \\
2. & 0,4048 & 0,2792 & 1,258 & \\
3. & 0,6538 & 0,4384 & 1,220 & 1,260 \\
4. & 0,3944 & 0,2760 & 1,274 &
\end{tabular}


Durch Zersetzen des Kalkniederschlages mit Ammoniumcarbonat wurden in Versuch 1: $0,0650 \mathrm{~g}$, in Versuch 2: 0,0596 g Dulcit regeneriert.

Diese Versuche zeigen, daß die mehrwertigen Alkohole 1 Molekül und mehr Kohlensäure zu binden vermögen. Die höchste Zahl, 1,415, wurde beim Mannit gefunden. Während der Unterschied zwischen dem Verhalten der einwertigen Alkohole und des zweiwertigen sehr bedeutend ist - Äthylalkohol und seine höheren Homologen gaben als Quotienten ca. 0,2 , Äthylenglykol 1,05 -, steigt das Vermögen, Kohlensäure $\mathrm{zu}$ binden, bis $\mathrm{zu}$ den sechswertigen Alkoholen nur wenig an.

\section{G. Zuckerarten.}

\section{Pentosen.}

\section{1-Arabinose.}

$0,1371 \mathrm{~g}$ bei $105^{\circ}$ bis zum konstanten Gewicht getrocknet im $100 \mathrm{ccm}$-Maßkolben gelöst und aufgefüllt. Nach 1,5 Stunden polarisiert.

$\alpha=+0,57^{\circ} \cdot 1=4$ T. $23^{\circ} \cdot \alpha_{[\mathrm{D}]}$ gefunden: $+103,94^{\mathrm{n}}$. Molekulargewicht: 150 .

Filtrat vom Kalkniederschlag auf $200 \mathrm{ccm}$ aufgefüllt, hiervon je $100 \mathrm{ccm}$ zur Polarisation und zur Bestimmung des $\mathrm{CaCO}_{3}$ verwendet. $1=4 \alpha_{[\mathrm{D}]}=104,4^{\circ}$.

\begin{tabular}{c|c|c|c|c|c}
\hline$\alpha$ & $\begin{array}{c}\text { der verbr. } \\
\text { Essigsäure } \\
\text { ccm }\end{array}$ & $\begin{array}{c}\text { Arabinose } \\
\mathrm{g}\end{array}$ & $\begin{array}{c}\mathrm{CaCO}_{3} \\
\mathrm{~g}\end{array}$ & Quotient & $\begin{array}{c}\text { Quotient } \\
\text { im Mittel }\end{array}$ \\
\hline 1,10 & 9,0 & 0,2882 & 0,0820 & 0,427 & 0,434 \\
1,30 & 9,15 & 0,3397 & 0,1038 & 0,441 &
\end{tabular}

2. Xylose.

$0,2790 \mathrm{~g}$ bei $100-110^{\circ}$ bis zum konstanten Gewicht getrocknete Substanz zu $100 \mathrm{ccm}$ gelöst. Nach 5 Stunden polarisiert.

$$
\alpha=0,22^{\circ} \cdot 1=4 \mathrm{~T} .=22^{\circ} \cdot \alpha_{[\mathrm{D}]} \text { gefunden: } 19,71^{\circ} .
$$


Molekulargewicht: 150. $\alpha_{[\mathrm{D}]}=19,39^{\circ} .1=4$. Filtrat vom Kalkniederschlag auf $200 \mathrm{ccm}$ aufgefüllt.

\begin{tabular}{c|c|c|c|c|c|c}
\hline \hline & $\begin{array}{c}\text { der verbr. } \\
\text { Essigsäure } \\
\mathrm{ccm}\end{array}$ & $\begin{array}{c}\text { Xylose } \\
\mathrm{g}\end{array}$ & $\begin{array}{c}\mathrm{CaCO}_{3} \\
\mathbf{g}\end{array}$ & Quotient & $\begin{array}{c}\text { Quotient } \\
\text { im Mittel }\end{array}$ \\
\hline $\mathbf{1 .}$ & 0,32 & 7,2 & 0,4423 & 0,0825 & 0,305 & 0,305 \\
$\mathbf{2 .}$ & 0,25 & 8,52 & 0,3601 & 0,0729 & 0,304 &
\end{tabular}

II. Hexosen.

\section{Traubenzucker.}

$1,2250 \mathrm{~g}$ des Hydrates zu $50 \mathrm{ccm}$ gelöst; 8 Stunden stehen gelassen. $\alpha=4,79^{\circ} .1=4 . \alpha_{[D]}$ des Hydrates $=48,36^{\circ}$ für wasserfreien Traubenzucker $=52,6^{\circ}$.

Molekulargewicht $=180$.

Filtrat vom Kalkniederschlag auf $200 \mathrm{ccm}$ aufgefüllt. $\alpha_{[D]}=53^{\circ} . \quad l=4$.

\begin{tabular}{c|c|c|c|c|c}
\hline \hline$\alpha$ & $\begin{array}{c}\text { Essigsäure } \\
\text { ccm }\end{array}$ & $\begin{array}{c}\text { Trauben- } \\
\text { zucker } \\
\mathbf{g}\end{array}$ & $\begin{array}{c}\mathrm{CaCO}_{8} \\
\mathbf{g}\end{array}$ & Quotient & $\begin{array}{c}\text { Quotient } \\
\text { im Mittel }\end{array}$ \\
\hline 1,36 & 4,65 & 0,6713 & 0,2018 & 0,543 & \\
1,65 & 12,05 & 0,8720 & 0,2004 & 0,423 & 0,485 \\
1,53 & 6,08 & 0,7710 & 0,2082 & 0,440 & \\
\end{tabular}

2. Galaktose.

$0,6056 \mathrm{~g}$ über Schwefelsäure bis zum konstanten Gewicht getrocknete Substanz in $8 \mathrm{ccm}$ Wasser gelöst, nach 7 Stunden auf $50 \mathrm{ccm}$ aufgefüllt. $\alpha=1,96^{\circ} . \mathrm{l}=2 . \mathrm{T} .=20^{\circ} . \alpha_{[\mathrm{D}]}: 80,91^{\circ}$.

Molekulargewicht $=180 . \alpha_{[D]}=80,3^{\circ}$.

Im ersten Versuche wurde das Filtrat vom Kalkniederschlage auf $200 \mathrm{ccm}$, im zweiten und dritten auf $100 \mathrm{ccm}$ aufgefüllt, im ersten Versuche je $100 \mathrm{ccm}$, im zweiten und dritten Versuche je $50 \mathrm{ccm}$ zur Bestimmung von $\alpha$ und Calciumcarbonat verwendet. 


\begin{tabular}{|c|c|c|c|c|c|c|}
\hline & $\alpha$ & $\begin{array}{l}\text { Essig- } \\
\text { säure } \\
\text { ccm }\end{array}$ & $\begin{array}{c}\text { Galac- } \\
\text { tose } \\
\mathrm{g}\end{array}$ & $\begin{array}{c}\mathrm{CaCO}_{3} \\
\mathrm{~g}\end{array}$ & Quotient & $\begin{array}{l}\text { Quotient } \\
\text { im Mittel }\end{array}$ \\
\hline 1. & 2,34 & $\check{5}, 35$ & 0,7674 & 0,2524 & 0,594 & \\
\hline 2. & 0,78 & 3,6 & 0,2575 & 0,0946 & 0,6614 & 0,635 \\
\hline 3. & 0,79 & 3,7 & 0,2612 & 0,0938 & 0,648 & \\
\hline
\end{tabular}

\section{Lävulose.}

$1,3788 \mathrm{~g}$ über $\mathrm{H}_{2} \mathrm{SO}_{4}$ bis zum konstanten Gewicht getrocknete Substanz zu $50 \mathrm{ccm}$ gelöst und nach 1 Stunde polarisiert.

$$
1=4 . \quad \alpha=-10,15^{\circ} . \text { T. }=20^{\circ} . \alpha_{[D]}=-91,85^{\circ} .
$$

Molekulargewicht: $180^{\circ}$. In Versuch 1 und $2 \alpha_{[D]^{20}}=$ $-92,13^{\circ}$, in Versuch 3 und $4 \alpha_{[D]}{ }^{18}=-91,57^{\circ} . \quad 1=4$. Filtrat auf $200 \mathrm{ccm}$ aufgefüllt.

\begin{tabular}{l|c|c|c|c|c|c}
\hline \hline & $\alpha$ & $\begin{array}{c}\text { Essig- } \\
\text { säure } \\
\text { ccm }\end{array}$ & $\begin{array}{c}\text { Lävu- } \\
\text { lose } \\
\mathrm{g}\end{array}$ & $\begin{array}{c}\mathrm{CaCO}_{3} \\
\mathrm{~g}\end{array}$ & Quotient & $\begin{array}{c}\text { Quotient } \\
\text { im Mittel }\end{array}$ \\
\hline & & & & & & \\
1. & $-2,18$ & 9,45 & 0,6474 & 0,2214 & 0,614 & \\
2. & $-3,57$ & 6,30 & 1,0273 & 0,2260 & 0,400 & 0,497 \\
3. & $-2,25$ & 8,65 & 0,6674 & 0,1786 & 0,482 & \\
4. & $-2,25$ & 9,60 & 0,6674 & 0,1826 & 0,492 &
\end{tabular}

III. Biosen.

\section{Rohrzucker.}

$0,8308 \mathrm{~g}$ bei 100 bis $103^{\circ}$ getrocknete Substanz zu $40 \mathrm{ccm}$ gelöst. $\alpha=5,46^{\circ} . \mathrm{l}=4 . \alpha_{[\mathrm{D}]}=65,74^{\circ}$. Filtrat auf $200 \mathrm{ccm}$ aufgefüllt.

Die Konzentration der Rohrzuckerlösung wurde erstens durch direkte Polarisation der mit Essigsäure ganz schwach angesäuerten Lösung, zweitens durch Polarisation nach Inversion mit Salzsäure bestimmt; für $\alpha_{[D]^{20}}$ des Rohrzuckers wurde 66,41 ${ }^{0}$, für $\alpha_{[D]^{20}}$ des Invertzuckers $19,657^{\circ}$ in Rechnung gesetzt. $l=4$.

Molekulargewicht des Rohrzuckers: 342, das dem Drehungsvermögen $\alpha_{[\mathrm{D}]^{20}}=19,657^{\circ}$ entsprechende des invertierten Zuckers $=360$. 


\begin{tabular}{|c|c|c|c|c|c|c|c|c|c|}
\hline $\mathrm{Nr}$. & $\begin{array}{c}\alpha_{1} \\
\text { durch } \\
\text { direkte } \\
\text { Polari- } \\
\text { sation }\end{array}$ & $\begin{array}{l}\text { Essig- } \\
\text { säure } \\
\mathrm{ccm}\end{array}$ & $\begin{array}{c}\alpha_{2} \\
\text { nach } \\
\text { Inver- } \\
\text { sion }\end{array}$ & $\begin{array}{c}\text { Zur In- } \\
\text { version } \\
\text { ver- } \\
\text { braucht } \\
\mathrm{HCl} \\
\mathrm{ccm}\end{array}$ & $\begin{array}{c}\text { Rohr- } \\
\text { zucker } \\
\text { aus } \alpha_{1} \\
\text { be- } \\
\text { rechnet } \\
\text { g }\end{array}$ & $\begin{array}{c}\text { Rohr- } \\
\text { zucker } \\
\text { aus } \alpha_{2} \\
\text { be- } \\
\text { rechnet } \\
\text { g }\end{array}$ & $\begin{array}{c}\mathrm{CaCO}_{3} \\
\mathrm{~g}\end{array}$ & $\begin{array}{l}\text { Quo- } \\
\text { tient } \\
\text { aus } \alpha_{1}\end{array}$ & $\begin{array}{l}\text { Quo- } \\
\text { tient } \\
\text { aus } \alpha_{2}\end{array}$ \\
\hline 1 & 1,18 & 2,1 & -0.42 & 5 & 0,4535 & 0,5591 & 0,1630 & 1,226 & 1,000 \\
\hline 2 & 2,04 & 3,2 & $-0,67$ & 5 & 0,8432 & 0,8904 & 0,2378 & 0,967 & 0,915 \\
\hline 3 & 3,39 & 3,25 & $-0,97$ & 5 & 1,3580 & 1,2885 & 0,3927 & 0,990 & 1,045 \\
\hline 4 & 2,00 & 3,3 & $|-0,52|$ & 5 & 0,8025 & 0,0911 & 0,2810 & 1,201 & 1,391 \\
\hline \multicolumn{8}{|c|}{ Quotienten im Mittel. . . } & 1,096 & 1,088 \\
\hline
\end{tabular}

\section{Milchzucker.}

$1,1528 \mathrm{~g}$ bei 100 bis $105^{\circ}$ getrocknete Substanz zu $40 \mathrm{ccm}$ gelöst, nach 24 Stunden polarisiert. $l=4 . \alpha=6,15^{\circ} . \alpha_{[\mathrm{D}]}=53,35^{\circ}$.

Molekulargewicht 360. $\quad \mathrm{l}=4 . \alpha_{[\mathrm{D}]}=52,53^{\circ}$ zur Berechnung genommen. Filtrat vom Kalkniederschlage auf $200 \mathrm{ccm}$ aufgefüllt.

\begin{tabular}{l|c|c|c|c|c|c}
\hline & $\alpha$ & $\begin{array}{c}\text { Essigsäure } \\
\text { ccm }\end{array}$ & $\begin{array}{c}\text { Milch- } \\
\text { zucker } \\
\text { g }\end{array}$ & $\begin{array}{c}\mathrm{CaCO}_{8} \\
\mathbf{g}\end{array}$ & Quotient & $\begin{array}{c}\text { Quotient } \\
\text { im Mittel }\end{array}$ \\
\hline $\mathbf{1 .}$ & 1,23 & 4,65 & 0,6117 & 0,1613 & 0,947 & \\
$\mathbf{2 .}$ & 1,31 & 4,05 & 0,6487 & 0,2026 & 1,127 & \\
$\mathbf{3 .}$ & 1,11 & 5,60 & 0,5570 & 0,1568 & 1,012 & \multirow{2}{*}{1,090} \\
$\mathbf{4 .}$ & 1,20 & 5,20 & 0,6008 & 0,2192 & 1,310 & \\
$\mathbf{5 .}$ & 1,80 & 2.70 & 0,8798 & 0,2090 & 1,102 & \\
6. & 1,72 & 4,00 & 0,8513 & 0,2460 & 1,042 &
\end{tabular}

\section{Maltose.}

$0,2375 \mathrm{~g}$ über $\mathrm{H}_{2} \mathrm{SO}_{4}$ bis zum konstanten Gewicht getrocknete Substanz zunächst mit $5 \mathrm{ccm}$ Wasser gelöst, nach 6 Stunden auf $100 \mathrm{ccm}$ aufgefüllt.

$$
\alpha=1,24^{\circ} . \quad l=4 . \quad \text { T. }=22^{\circ} . \alpha_{[\mathrm{D}]}=136,26^{\circ} .
$$

Molekulargewicht $360^{\circ}$.

Filtrat auf $200 \mathrm{ccm}$ aufgefüllt. 
$\alpha_{[D]}{ }^{22}$ für Versuch 1: $138,26^{0} . \alpha_{[D]^{24}}{ }^{24}$ für Versuch 2: $138,075^{0}$. $\alpha_{[D]^{23}}$ für Versuch 3: $138,17^{\circ} . \quad \mathrm{l}=4$.

\begin{tabular}{c|c|c|c|c|c|c}
\hline \hline & $\alpha$ & $\begin{array}{c}\text { Essigsäure } \\
\mathrm{ccm}\end{array}$ & $\begin{array}{c}\text { Maltose } \\
\mathrm{g}\end{array}$ & $\begin{array}{c}\mathrm{CaCO}_{3} \\
\mathrm{~g}\end{array}$ & Quotient & $\begin{array}{c}\text { Quotient } \\
\text { im Mittel }\end{array}$ \\
\hline 1. & 3,11 & 5,3 & 0,5922 & 0,1604 & 1,103 & \\
2. & 1,69 & 8,1 & 0,3310 & 0,0928 & 1,000 & 1,109 \\
3. & 1,73 & 6,7 & 0,3338 & 0,1114 & 1,225 &
\end{tabular}

Im Mittel wurden demnach gefunden für :

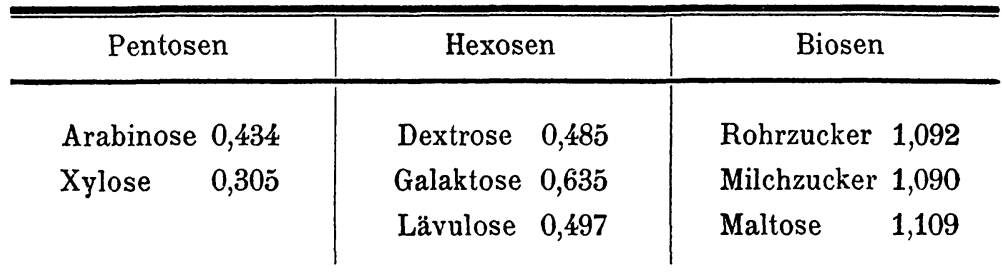

Die Pentosen binden also weniger als ein halbes Molekül Kohlensäure, von den Hexosen die Dextrose und die Lävulose ziemlich genau $1 / 2$ Molekül, die Galactose etwas mehr und die Biosen sehr übereinstimmend 1,1 Molekül Kohlensäure.

D. Oxysäuren.

1. Oxyessigsäure.

Molekulargewicht 76. Fp. gefunden $77,3^{\circ}$.

\begin{tabular}{l|c|c|c|c}
\hline \hline & $\begin{array}{c}\text { Essigsäure, } \\
\mathrm{g}\end{array}$ & $\begin{array}{c}\mathrm{CaCO}_{3} \\
\mathrm{~g}\end{array}$ & Quotient & Quotient im Mittel \\
\hline & & 0,1146 & 0,086 & \\
2. & 1,0128 & 0,1628 & 0,118 & 0,109 \\
3. & 1,0487 & 0,1100 & 0,141 & \\
4. & 0,0930 & 0,1232 & 0,091 &
\end{tabular}

Aus dem Kalkniederschlage des Versuches 3 wurde nur $0,0104 \mathrm{~g}$, aus dem des Versuches 4 nur 0,0106 g Oxyessigsäure regeneriert. Selbst wenn diese geringen Mengen nicht als Salze des Kohlensäurederivates in den Niederschlägen vor- 
handen waren und von den angewandten Substanzmengen abzuziehen wären, würde der Quotient kaum geändert.

\section{Gärungsmilchsäure.}

Verwendet wurde das bei $100-110^{\circ}$ bis zum konstanten Gewichte getrocknete Calciumsalz.

Reinheitsprüfung: $0,1863 \mathrm{~g}$ bei $100-110^{\circ}$ bis zum konstanten Gewicht getrocknetes Salz gaben $0,0484 \mathrm{~g} \mathrm{CaO}$.

Ca gefunden: 18,57\% ; berechnet: 18,35\%.

Molekulargewicht: $\mathrm{CH}_{3} \cdot \mathrm{CH} \cdot \mathrm{OH} \cdot \mathrm{COO}$ ca.: 109.

\begin{tabular}{|c|c|c|c|c|}
\hline & $\begin{array}{c}\text { Calciumlactat } \\
\mathrm{g} \\
\end{array}$ & $\begin{array}{c}\mathrm{CaCO}_{3} \\
\mathrm{~g}\end{array}$ & Quotient & Quotient im Mittel \\
\hline 1. & 0,5714 & 0,1354 & 0,257 & \multirow{19}{*}{0,22} \\
\hline 2. & 0,6195 & 0,1495 & 0,265 & \\
\hline 3. & 0,8388 & 0,1170 & 0,150 & \\
\hline 4. & 0,7922 & 0,1866 & 0,257 & \\
\hline 5. & 0,8665 & 0,1764 & 0,222 & \\
\hline 6. & 0,5000 & 0,1062 & 0,232 & \\
\hline 7. & 0,5000 & 0,0940 & 0,205 & \\
\hline 8. & 0,9146 & 0,1650 & 0,197 & \\
\hline 9. & 0,9564 & 0,1848 & 0,210 & \\
\hline 10. & $0, \check{5000}$ & 0,690 & 0,153 & \\
\hline 11. & 1,0166 & 0,1882 & 0,203 & \\
\hline 12. & 1,2658 & 0,2372 & 0,205 & \\
\hline 13. & 0,4796 & 0,1132 & 0,271 & \\
\hline 14. & 0,8786 & 0,1821 & 0,226 & \\
\hline 15. & 1,2736 & 0,3358 & 0,287 & \\
\hline 16. & 0,5112 & 0,1042 & 0,221 & \\
\hline 17. & 1,6124 & 0,2294 & 0,155 & \\
\hline 18. & 1.9061 & 0,3170 & 0,165 & \\
\hline 19. & 2,6656 & 0,6609 & 0,270 & \\
\hline
\end{tabular}

Die Substanzmengen waren in je $50 \mathrm{ccm}$ gelöst worden.

\section{Paramilchsäure.}

Die Paramilchsäure wurde aus Fleischextrakt «mit der Flagge» dargestellt. Verwendet wurde auch hier das bei 100 bis $110^{\circ}$ bis zum konstanten Gewichte getrocknete Calciumsalz. 
Reinheitsprüfung: $0,3495 \mathrm{~g}$ bei $100-110^{\circ}$ bis zum konstanten Gewicht getrockneter Substanz gaben $0,0911 \mathrm{~g} \mathrm{CaO}$.

Ca gefunden: $18,62 \%$; berechnet: $18,35 \%$.

\begin{tabular}{c|c|c|c|c}
\hline & $\begin{array}{c}\text { Calciumparalactat } \\
\mathrm{g}\end{array}$ & $\begin{array}{c}\mathrm{CaCO}_{8} \\
\mathrm{~g}\end{array}$ & Quotient & Quotient im Mittel \\
\hline 1. & 0,3766 & 0,0970 & 0,281 & 0,277 \\
2. & 0,6978 & 0,1756 & 0,274 &
\end{tabular}

Die Substanzmengen waren in je $50 \mathrm{ccm}$ Wasser gelöst worden.

4. Oxyisobuttersäure.

$\left(\mathrm{CH}_{3}\right)_{2} \cdot \mathrm{C}(\mathrm{OH}) \cdot \mathrm{COOH}$.

Molekulargewicht: 104. Fp. $78^{\circ}$.

\begin{tabular}{c|c|c|c|c}
\hline & $\begin{array}{c}\text { Oxyisobuttersäure } \\
\mathrm{g}\end{array}$ & $\begin{array}{c}\mathrm{CaCO}_{8} \\
\mathrm{~g}\end{array}$ & Quotient & Quotient im Mittel \\
\hline 1. & 0,5094 & 0,0128 & 0,027 & 0,029 \\
2. & 0,5220 & 0,0160 & 0,0318 &
\end{tabular}

Von den vier untersuchten Oxysäuren besitzen die 0xyessigsäure, Gärungsmilchsäure und Paramilchsäure ein ausgeprägtes Vermögen, Kohlensäure bei Gegenwart von Kalkhydrat zu addieren; bei den Milchsäuren ist dieses Vermögen mehr als doppelt so groß als bei der Oxyessigsäure; aber auch bei diesen kommt unter den betreffenden Versuchsbedingungen nur etwa 1 Molekül Kohlensäure auf 4 Moleküle Oxysäure. Die Oxyisobuttersäure, bei der die Hydroxylgruppe sich an einem tertiären Kohlenstoffatome befindet, reagiert fast gar nicht. Wir sehen hier das gleiche Verhalten wie bei dem tertiären Butylalkohol.

\section{Die Erklärung der Hydroxylkohlensäurereaktion.}

Zunächst fragt es sich:

Was entsteht bei der Reaktion?

Die bisher mitgeteilten Tatsachen zeigen, daß die Gegenwart von Hydroxylkörpern der Fettreihe bewirkt, daß, wenn man Kohlensäure in Kalkmilch bei $0^{\circ}$ einleitet und filtriert, das Filtrat beim Stehen bei gewöhnlicher Temperatur oder beim 
Erwärmen Calciumcarbonat abscheidet, und daß die Menge dieses Calciumcarbonates für jeden Hydroxylkörper innerhalb gewisser Grenzen konstant ist. Für die Aminokörper ist durch Reindarstellung der bei der entsprechendenReaktion entstehenden Verbindungen der Beweis geliefert, daß die Kohlensäure unter Bildung von Carbonsäuren, der Carbaminosäuren, addiert wird. Findet nun bei den Hydroxylkörpern die analoge Reaktion statt? Ist dies der Fall, so entstehen die Salze der Hydroxylcarbonsäuren; z. B.:

\section{$\mathrm{CH}_{3} \mathrm{OH} \longrightarrow \mathrm{CH}_{3} \mathrm{OCOO}$ ca.}

Die Reaktion verläuft in sehr verdünnten Lösungen, in $0,1 \%$ igen und verdünnteren. Schlüsse aus der Art der Reaktion der Einwirkung von Kohlensäure z. B. auf methylalkoholische Lösungen von Baryumoxyd ${ }^{1}$ ) auf die Hydroxylkohlensäurereaktion $\mathrm{zu}$ ziehen, ist daher nicht angängig. ${ }^{2}$ )

In unserem Falle ist es ausgeschlossen, daß die Reaktion auf der Bildung von kolloidem Calciumcarbonat beruht. Denn wenn man bei Ausführung der Reaktion einen großen Überschuß von Calciumoxydhydrat in der Lösung vermeidet, so wird die Reaktion des Filtrates, welche ursprünglich stark alkalisch ist, sauer, wenn sich beim Stehen oder Erwärmen Calciumcarbonat abscheidet. Hierfür gibt es keine Erklärung, wenn das abgeschiedene Calciumcarbonat aus kolloidem Calciumcarbonat entstanden wäre. Wohl aber findet der Übergang der alkalischen Reaktion in die saure seine volle Erklärung, wenn bei der Hydroxylkohlensäurereaktion hydroxylkohlensaure Salze entstehen. Denn wenn sich diese beim Stehen oder Erwärmen ihrer wässerigen Lösungen zersetzen, so bildet sich freie Kohlensäure und die ursprünglich alkalische Reaktion geht in die saure über:

$$
\mathrm{CH}_{3} \mathrm{OCOO}-\mathrm{Ca}+\mathrm{H}_{2} \mathrm{O}=2 \mathrm{CH}_{3} \mathrm{OH}+\mathrm{CO}_{3} \mathrm{Ca}+\mathrm{CO}_{2} \text {. }
$$

1) C. Neuberg u. E. Neimann, Zeitschr. f. Biochemie, Bd. I, S. 166.

C. Neuberg u. B. Rewald, ebenda, Bd. IX, S. 536.

2) Ebenso hat die relative Löslichkeit von Calciumcarbonat z. B. in konzentrierten Zuckerlösungen mit der Hydroxylkohlensäurereaktion nichts $\mathrm{zu}$ tun. 
Dieser Übergang der alkalischen Reaktion in die saure wird bei allen Hydroxylkörpern beobachtet. (Bei der Bestimmung des Quotienten ist es daher nötig, in der Lösung für einen entsprechenden Gehalt an Calciumoxydhydrat, welcher die Kohlensäure aufnimmt, zu sorgen.) Schon deshalb ist nicht zu zweifeln, daß bei der Hydroxylkohlensäurereaktion hydroxylkohlensaure Salze entstehen, z. B.

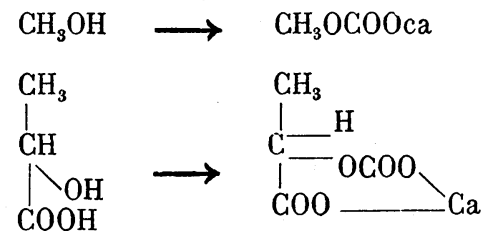

Diese Anschauung wird durch folgende Versuche weiter gestützt.

\section{Versuche zur Darstellung der Calciumsalze von Glycerincarbonsäure und Äthylenglykolcarbonsäure.}

Es sollte versucht werden, analog den Salzen der Carbaminosäuren solche der Hydroxylkohlensäure darzustellen. Bei den Versuchen mit primären Alkoholen in verdünnten wässerigen Lösungen gelang es nicht, durch Alkohol Niederschläge zu erzielen. Hingegen glückte es beim Glycerin und Äthylenglykol.

1. Glycerincarbonsaures Calcium.

$\mathrm{W}^{\top}$ egen der leichten Zersetzlichkeit der hydroxylkohlensauren Calciumsalze nach Abfiltrieren deren Lösungen vom Calciumcarbonat wurde folgendermaßen verfahren: Die Reaktion wurde wie bei Bestimmung des Quotienten in schwacher Kältemischung ausgeführt, die Mischung ebenso abgesaugt und das Filtrat unmittelbar in absolutem Alkohol, der auf mehrere Grade unter 0 durch eine Kältemischung gehalten wurde, tropfen gelassen. Der entstandene Niederschlag wurde in eine Kältemischung abgesaugt, mit in der Kältemischung gekühltem absoluten Alkohol nachgewachen und über Schwefelsäure im Vakuum getrocknet.

Zunächst überzeugten wir uns durch 2 Versuche mit $6 \%$ iger und 25\% iger Glycerinlösung, daß unter gleichen Bedingungen, wie bei den späteren Versuchen, nur mit Hinweglassung der Kohlensäure, durch Alkohol aus der mit Kalkhydrat 
gesättigten wässerigen Lösung des Glycerins keine Fällung entsteht. Diese Versuche beweisen, daß die nach Einleiten von Kohlensäure aus dem Filtrate durch Alkohol gefällten Niederschäge nicht etwa Glycerincalciumverbindungen enthalten können.

I. Qualitative Untersuchung der glycerincarbonsauren Salze.

Die Calciumsalze der Glycerincarbonsäuren werden, wenn sie beim Einbringen in den Exsikkator eine geringe Menge Feuchtigkeit anzuziehen Gelegenheit haben, beim Stehen über Schwefelsäure glasig, beim Zerkleinern entstehen dann Splitter, die durchsichtig sind und bei oberflächlicher mikroskopischer Betrachtung für Krystalle gehalten werden könnten. Sie sind aber unregelmäßig begrenzt; auch erweisen sie sich unter dem Polarisationsmikroskop als inaktiv.

Wird die Feuchtigkeit sorgfältigst ausgeschlossen, so erhält man das Salz in Form von Körnchen.

Beim Erhitzen im Glühröhrchen schwärzt sich das Salz unter Abscheidung von Kohle und entwickelt Akrolein. Hierdurch ist zunächst bewiesen, daß eine Glycerinverbindung vorliegt, welche, da sie unter gleichen Bedingungen, jedoch bei Abwesenheit von Kohlensäure nicht entsteht, unter Mitwirkung der Kohlensäure aus Glycerin entstanden ist. Die so untersuchten Calciumsalze waren aus 6- und 25\% iger Glycerinlösung dargestellt worden.

II. Quantitative Untersuchung der glycerincarbonsauren Salze.

Die Salze wurden aus einer 25\% igen wässerigen Glycerinlösung dargestellt.

Bei der Einwirkung von Kohlensäure auf Glycerin bei Gegenwart von Kalkhydrat können die Salze der Glycerinmonocarbonsäure, Glycerindicarbonsäure und Glycerintricarbonsäure entstehen.

I.

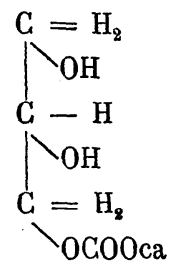

II.

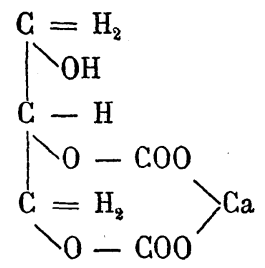

III.

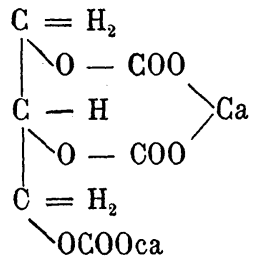


Die empirische Zusammensetzung dieser Salze entspricht folgenden Zahlen.

I.

$\begin{array}{lr}\text { C } & 30,97 \% \\ \mathrm{H} & 4,52 \% \\ \mathrm{C} a & 12,90 \%\end{array}$

II.

$27,52 \%$

$2,75 \%$

$18,35 \%$
III.

$25,62 \%$

$1,78 \%$

$21,35 \%$

Die bei verschiedenen Darstellungen erhaltenen analytischen Werte differieren unter einander.

Zunächst wurde an einem Präparate geprüft, ob, wie es geschehen müßte, wenn Calciumsalze eine der drei möglichen Glycerincarbonsäuren oder deren Gemenge entstanden waren, alles Calcium beim Erwärmen mit Wasser als Calciumcarbonat abgespalten wird.

a) Ca-Bestimmungen.

1. $0,2203 \mathrm{~g}$ über $\mathrm{H}_{2} \mathrm{SO}_{4}$ bis zum konstanten Gewicht getrockneter Substanz gaben $0,0501 \mathrm{~g} \mathrm{CaO}=16,25 \% \mathrm{Ca}$.

2. $0,2860 \mathrm{~g}$ über $\mathrm{H}_{2} \mathrm{SO}_{4}$ bis zum konstanten Gewicht getrockneter Substanz gaben $0,0652 \mathrm{~g} \mathrm{CaO}=16,32 \% \mathrm{Ca}$.

b) Bestimmung des als Calciumcarbonat abspaltbaren Calciums bei denselben Präparaten:

$0,1672 \mathrm{~g}$ über $\mathrm{H}_{2} \mathrm{SO}_{4}$ bis zum konstanten Gewicht getrockneter Substanz wurde auf dem Wasserbade mit ca $30 \mathrm{ccm}$ Wasser erwärmt, das abgespaltene Calciumcarbonat auf GoochTiegel filtriert, bei $120^{\circ}$ getrocknet.

Erhalten $0,0673 \mathrm{gr} \mathrm{CaCO}_{3}=16,10 \% \mathrm{Ca}$.

Das Calcium wird also vollständig als $\mathrm{CaCO}_{3}$ beim Erwärmen mit Wasser abgespalten.

Auch bei den folgenden Analysen sind die Substanzen über Schwefelsäure bis zum konstanten Gewichte getrocknet.

Bei den C- und H-Bestimmungen wurden die Substanzen erst für sich verbrannt und nach Wägung des Chlorcalciumrohrs nochmals nach Zugabe von Kaliumbichromat zu dem Schiffcheninhalt.

\section{Darstellung II.}

1. 0,2788 g Substanz gaben $0,0960 \mathrm{~g} \mathrm{H}_{2} \mathrm{O}$ und $0,2823 \mathrm{~g} \mathrm{CO}_{2}$.

$$
\mathrm{H}=3,80 \% ; \mathrm{C}=27,61 \% \text {. }
$$

2. $0,2730 \mathrm{~g}$ Substanz gaben $0,0960 \mathrm{~g} \mathrm{H}_{2} \mathrm{O}$ und $0,2786 \mathrm{~g} \mathrm{CO}_{2}$.

$$
\mathrm{H}=3,90 \% ; \mathrm{G}=27,83 \% \text {. }
$$

3. 0,3326 g Substanz gaben $0,0860 \mathrm{~g} \mathrm{Ca}$; $\mathrm{Ca}=18,46 \%$. 


\section{Darstellung III.}

1. 0,2168 g Substanz gaben $0,0728 \mathrm{~g} \mathrm{H}_{2} \mathrm{O}$ und $0,2284 \mathrm{~g} \mathrm{CO}_{2}$.

$$
\mathrm{H}=3,74 \% ; \mathrm{C}=28,74 \% \text {. }
$$

2. 0,1712 g Substanz gaben $0,0586 \mathrm{~g} \mathrm{H}_{\mathrm{r}} \mathrm{O}$ und $0,1816 \mathrm{~g} \mathrm{CO}_{2}$.

$$
\mathrm{H}=3,80 \% ; \mathrm{C}=28,91 \% \text {. }
$$

3. 0,2086 g Substanz gaben $0,0700 \mathrm{~g} \mathrm{H}_{2} \mathrm{O}$ und 0,2204 $\mathrm{g} \mathrm{CO}_{2}$.

$$
\mathrm{H}=3,74 \% ; \mathrm{C}=28,81 \% \text {. }
$$

4. 0,1880 g Substanz gaben $0,0446 \mathrm{~g} \mathrm{CaO} ; \mathrm{Ca}=16,96 \%$.

5. 0,2445 > \ \0,0586 > > $>17,12 \%$.

$6.0,2206$ > > 0,0530 > > $>17,18 \%$.

Die Analysenwerte der Darstellung II stimmen mit den für das Calciumsalz der Glycerindicarbonsäure berechneten, mit Ausnahme des Wasserstoffs, der ca. 1\% zu hoch gefunden wurde, überein (berechnet: $\mathrm{C}=27,52 \% ; \mathrm{Ca}=18,35 \% ; \mathrm{H}=2,75 \%$ ). Dies kann durch die große Hygroskopizität der Substanz, welche beim Einbringen des Schiffchens in das Verbrennungsrohr Wasser anzieht, erklärt werden. Die Werte der Darstellung III entfernen sich durchweg um ca. 1\% von den berechneten.

Man kann aus den Ergebnissen der Analysen also nicht den sicheren Schluß ziehen, daß das Calciumsalz der Glycerindicarbonsäure entsteht, sondern muß mit der Möglichkeit rechnen, daß ein Gemenge der Calciumsalze zweier oder der drei möglichen Glycerincarbonsäuren vorliegt.

\section{2. Äthylenglykolcarbonsaures Calcium.}

Zunächst wurde wieder geprüft, ob nicht etwa Äthylenglykolcalcium ausgeschieden würde, wenn unter den bei den Versuchen mit Kohlensäure einzuhaltenden Bedingungen wässerige Lösungen von Äthylenglykol ohne Einleiten von Kohlensäure mit Kalkmilch geschüttelt, und das Filtrat in Alkohol gesaugt wurde. Zu dem Zwecke wurden 1) $2 \mathrm{~g}$ Glykol in $15 \mathrm{ccm}$ Wasser, 2) $7 \mathrm{~g}$ Glykol in $25 \mathrm{ccm}$ Wasser, 3) $15 \mathrm{~g}$ Glykol in $50 \mathrm{ccm}$ Wasser gelöst, in der Kältemischung mit Kalkmilch geschüttelt und in je $100 \mathrm{ccm}$ 99\%igen Alkohol, der durch eine Kältemischung auf einige Grade unter $0^{\circ}$ gehalten, wurde, abgesaugt. Bei keinem dieser Versuche fand eine Ausscheidung im Alkohol statt. Es war demnach nicht zu befürchten, daß, wenn nach Einleiten von Kohlen- 
säure eine Ausscheidung unter sonst gleichen Verhältnissen erhalten wurde, diese zum Teil aus Äthylenglykolcalcium bestünde.

Schon $4 \%$ ige wässerige Lösungen gaben nach Behandeln mit Kohlensäure etc. Fällungen im Alkohol. $\mathrm{Zu}$ dem zu den Analysen verwendeten Präparate waren, da die Ausbeuten in jedem einzelnen Falle nur gering waren, die Fällungen von mehreren Darstellungen, bei denen von 30 - bis $50 \%$ igen Glykollösungen ausgegangen war, vereinigt.

Das durch Alkohol ausgeschiedene Salz wurde schnell abgesaugt, mit 99\% igem Alkohol gewaschen und im Vakuum über Schwefelsäure getrocknet. Das Salz ist sehr hygroskopisch, wird, wenn es etwas Wasser anzieht, glasig und in kleinen Stücken durchsichtig. Krystallformen oder Doppelbrechung ließen sich nicht nachweisen.

\section{Analysen.}

$\mathrm{Zu}$ den Analysen wurde das Salz über Schwefelsäure im Vakuum bis zum konstanten Gewichte getrocknet. Bei den CBestimmungen wurde wie bei den Analysen des Salzes der Glycerincarbonsäure mit Kaliumbichromat nachverbrannt.

I. $0,1248 \mathrm{~g}$ Substanz gaben $0,0318 \mathrm{~g} \mathrm{H}_{2} \mathrm{O}$ und $0,1184 \mathrm{~g} \mathrm{CO}_{2}$

II. 0,1589 > > > 0,0380 > > 0,1486 , ,

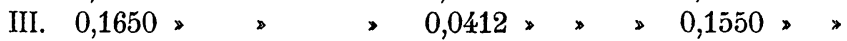

IV. $0,1124,>\quad>0,0341$ $>\mathrm{CaO}$.

$\%$ gefunden:

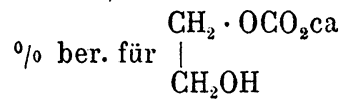
für $\underset{\mathrm{CH}_{2} \mathrm{OCO}_{2} \mathrm{Ca}}{\mathrm{CH}_{2} \cdot \mathrm{OCO}_{2} \mathrm{Ca}}$

$\mathrm{C}=25,88 ; 25,49 ; 25,63$

28,80

25,53

$\mathrm{H}=2,80 ; 2,64 ; 2,77$

$\mathrm{Ca}=21,62$

4,00

2,13

16,00

21,27

Die gefundenen Zahlen stimmen also recht gut mit den für das Calciumsalz der Glykoldicarbonsäure berechneten überein; mit Ausnahme des Wasserstoffes, der etwas zu hoch gefunden wurde, was bei der sehr hygroskopischen Substanz nicht wundernimmt. Sie würden sich weder mit denen eines Gemenges der Calciumsalze der Mono- und Dicarbonsäure, noch mit denen des Salzes der Monocarbonsäure $+\mathrm{xH}_{2} \mathrm{O}$ vereinigen lassen, sodaß es keinem Zweifel unterliegt, daß durch Einwirkung von Kohlensäure auf Äthylenglykol bei Gegen- 
wart von Kalkhydrat das Calciumsalz der Äthylenglykoldicarbonsäure entsteht. Somit ist in diesem Falle die Reindarstellung eines hydroxylkohlensauren Salzes geglückt.

Nachdem festgestellt wurde, daß bei der Hydroxylkohlensäurereaktion Salze der Hydroxylcarbonsäuren entstehen, ist die Frage zu diskutieren:

Wie verläuft die Reaktion?

Verliefe die Reaktion quantitativ, mit anderen Worten, würde z. B. vom Methylalkohol 1 Molekül Kohlensäure gebunden, so stünde der Annahme lediglich folgenden Reaktionsverlaufes nichts entgegen:

I. $\mathrm{CH}_{3} \mathrm{OH}+\mathrm{CaOH}+\mathrm{CO}_{2}=\mathrm{CH}_{2} \cdot \mathrm{OCO}_{2} \mathrm{Ca}+\mathrm{H}_{2} \mathrm{O}$.

So aber wird nur etwa ein halbes Molekül Kohlensäure gebunden. Da nicht anzunehmen ist, daß eine andere Reaktion als die Bildung des Salzes der Hydroxylcarbonsäure stattfindet, so ist der Umstand, daß ein zwischen 0 und 1 liegender Wert erhalten wird, nur dadurch zu erklären, daß ein Gleichgewichtszustand eintritt. Der Vorgang muß also durch eine umkehrbare Gleichung ausdrückbar sein.

II. $\mathrm{CH}_{3} \mathrm{OH}+\mathrm{CO}_{3} \mathrm{Ca} \rightleftarrows \mathrm{CH}_{3} \cdot \mathrm{OCO}_{2} \mathrm{ca}+\mathrm{caOH}$.

Diese an und für sich mögliche Gleichung entspricht jedoch nicht den Tatsachen. Denn wird frischgefälltes Calciumcarbonat mit der Lösung von Methylalkohol in Wasser bei $0^{0}$ geschüttelt, so bildet sich kein hydroxylkohlensaures Salz.

Versuch: $25 \mathrm{ccm}$ Kalkmilch wurde nach Zusatz einiger Tropfen der Lösung von Phenolphthalein in Kalkwasser mit Kohlensäure gesättigt, die Mischung 4 Stunden auf der Maschine geschüttelt, um noch nicht in Reaktion getretene Partikelchen von Kalkhydrat in Lösung zu bringen; hierauf wurde wieder Kohlensäure eingeleitet und wieder geschüttelt, in der Kältemischung abgekühlt, bis etwas Eis krystallisierte, dazu 0,2 $\mathrm{g}$ Methylalkohol in $50 \mathrm{ccm}$ Wasser gegeben, in der Kältemischung unter häufigem Schütteln 40 Minuten gelassen. Nach Absaugen gab das Filtrat beim Kochen keine Spur einer Abscheidung. Dieser Versuch wurde mehrere Male mit dem gleichen Erfolge wiederholt. Es bildet sich also aus Calciumcarbonat und Methylalkohol kein hydroxylkohlensaures Salz. 
Wohl aber erfolgte die Reaktion prompt, wenn zu der Mischung von Calciumcarbonat und der Lösung von $0,2 \mathrm{~g}$ Methylalkohol in $50 \mathrm{ccm}$ Wasser Kalkmilch gegeben wurde.

Versuche: In 2 hintereinander angestellten Versuchen wurden je $25 \mathrm{ccm}$ auf $0^{\circ}$ abgekühlter Kalkmilch nach Zusatz einiger Tropfen einer Lösung von Phenolphthalein in Kalkwasser mit Kohlensäure neutralisiert, darauf wurde 0,2 g Methylalkohol in $50 \mathrm{ccm}$ Wasser und $25 \mathrm{ccm}$ Kalkmilch gegeben und unter Kühlung in schwacher Kältemischung unter häufigem Umschütteln 40 Minuten stehen gelassen. Es wurden bei Bestimmung des Quotienten erhalten:

$\begin{array}{ccc} & \mathrm{g} \mathrm{CaCO}_{3} & \text { Quotient } \\ \text { I. } & 0,0662 & 0,106 \\ \text { II. } & 0,0778 & 0,123\end{array}$

Man sieht also, daß die Reaktion auch unter diesen Bedingungen vonstatten geht. Die Löslichkeit des Calciumcarbonates in Wasser, welche bei $18-25^{\circ} 0,0013 \mathrm{~g}: 100$ beträgt, ${ }^{1}$. gestattet immerhin die Annahme des Bestehens eines Gleichgewichtes.

Zum Stattfinden der Reaktion ist also die Gegenwart von Calciumoxydhydrat nötig. Dem Vorgange würde folgende Gleichung entsprechen.

III : $\mathrm{CH}_{3} \cdot \mathrm{OH}+\mathrm{CaOH}+\mathrm{CO}_{3} \mathrm{Ca} \rightleftarrows \mathrm{CH}_{3} \cdot \mathrm{OCO}_{2} \mathrm{Ca}+\mathrm{Ca}_{\mathrm{OH}}$

Die Tatsache, daß Calciumoxydhydrat zugegen sein muß, findet wohl am besten ihre Erklärung durch die Annahme, daß sich Methylalkoholcalcium bildet, das zwar in wässeriger Lösung stark hydrolysiert ist, dessen Hydrolyse aber durch den Überschuß von Kalkhydrat in der an diesem gesättigten Lösung zurückgedrängt wird. Die Gleichung würde dann folgendermaßen lauten:

$$
\text { IV: } \mathrm{CH}_{3} \cdot \mathrm{Oca}+\mathrm{CO}_{3} \mathrm{Ca}+\mathrm{H}_{2} \mathrm{O} \rightleftarrows \mathrm{CH}_{3} \mathrm{OCO}_{2} \mathrm{ca}+\mathrm{Ca}_{\mathrm{OH}}^{\mathrm{OH}}
$$

Aus den Gleichungen III resp. IV ersieht man, daß die Reaktion gar nicht durch die Gegenwart von Wasser gehemmt

1) W. Böttger, Qualitative Analyse vom Standpunkte der Ionenlehre, II. Aufl., S. 513. 
wird; es erklärt sich so die zunächst befremdende Tatsache, daß die Bildung der Hydroxylkohlensäuren in so weitem Umfange in so verdünnten wässerigen Lösungen erfolgt. Erst bei einer höheren Temperatur findet dann eine bei der niederen Temperatur nicht zur Geltung kommende andere Reaktion merklich statt, nämlich die Zersetzung der Salze der Hydroxylkohlensäuren durch Wasser nach folgender Gleichung:

$\mathrm{R} \cdot \mathrm{OCOOCa}+\mathrm{HOH}=\mathrm{R} \cdot \mathrm{OH}+\mathrm{CO}_{3} \mathrm{Hca}$.

Wir stellen uns also vor, daß sowohl die Reaktion I als III resp. IV vor sich geht.

Ganz dasselbe, was hier für die Hydroxylkohlensäurereaktion auseinander gesetzt ist, gilt mutatis mutandis für die Carbaminoreaktion. Auch hier wurde festgestellt, daß Glykokoll mit frischgefälltem Calciumcarbonat keine Spur glykokollcarbonsauren Calciums gibt, daß aber Calciumcarbonat + Kalkmilch + Glykokoll glykokollcarbonsaures Calcium gibt. 2 Versuche, bei denen entsprechend der früher ${ }^{1}$ ) gegebenen Vorschrift der Quotient der Carbaminoreaktion bestimmt wurde, nur mit dem Unterschiede, daß erst nach Zusatz von Kalkmilch zu dem aus Kalkmilch zuerst durch Kohlensäure dargestelltem Calciumcarbonat Glykokoll gegeben wurde und dann nicht wieder Kohlensäure eingeleitet wurde, gaben folgende Resultate:

$\begin{array}{cccc} & \text { Gefunden } \mathbf{g ~ C a C O} \text { Can }_{3}: & \text { Verbraucht } \mathbf{n} / \mathbf{1 0}-\mathrm{S} & \mathbf{x} \\ \text { I. } & 0,2193 & 49,0 & 2,3 \\ \text { II. } & 0,0641 & 17,8 & 2,8\end{array}$

Während bekanntlich beim Einleiten von Kohlensäure in die mit Kalkmilch versetzte Lösung des Glykokolls 1 Mol. desselben $1 \mathrm{Mol}$. $\mathrm{CO}_{2}$ aufnimmt, ist bei diesen Versuchen nur etwa die Hälfte des Glykokolls in das Salz der Glykokollcarbonsäure übergeführt worden. Jedenfalls geht die Reaktion auch hier lediglich bei Gegenwart von Glykokoll, Calciumcarbonat und Calciumoxydhydrat vonstatten und entspricht folgender Gleichung.

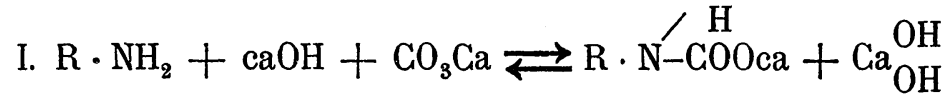

1) M. Siegfried u. C. Neumann, Diese Zeitschrift, Bd. LIV, S. 423. 
404 Siegfried und How wjanz, Über die Bindung von Kohlensäure usw.

Diese Erklärung der Hydroxylkohlensäure- und Carbaminoreaktion erfordert zur Prüfung noch weitere Versuche, welche demnächst ausgeführt werden sollen.

Jedenfalls steht fest, daß Aminosäuren und Hydroxylkörper Calciumcarbonat bei Gegenwart von Calciumoxydhydrat aufzulösen imstande sind, daß also Calciumcarbonat bei alkalischer Reaktion gelöst werden kann. Diese Tatsache erscheint uns als Grundphänomen biologische Bedeutung zu haben. Die Gegenwart von Amino- oder Hydroxylkörpern bewirkt bei alkalischer Reaktion dasselbe, was freie Säuren tun. Alkalische Flüssigkeiten, die Aminokörper oder Hydroxylkörper enthalten, können Calciumcarbonat auflösen und in gelöster Form transportieren. 